\title{
Clostridium difficile infection after cardiac surgery: Prevalence, morbidity, mortality, and resource utilization
}

\author{
Suresh Keshavamurthy, MBBS, MS, ${ }^{a}$ Colleen G. Koch, MD, MS, MBA, ${ }^{\text {b,c }}$ Thomas G. Fraser, MD, ${ }^{c, d}$ \\ Steven M. Gordon, MD, ${ }^{\mathrm{d}}$ Penny L. Houghtaling, MS, ${ }^{\mathrm{e}}$ Edward G. Soltesz, MD, MPH, ${ }^{\mathrm{a}}$ \\ Eugene H. Blackstone, MD, ${ }^{\mathrm{a}, \mathrm{e}}$ and Gösta B. Pettersson, $\mathrm{MD}, \mathrm{PhD}^{\mathrm{a}}$
}

\begin{abstract}
Objective: Despite increasing efforts to prevent infection, the prevalence of hospital-associated Clostridium difficile infections (CDI) is increasing. Heightened awareness prompted this study of the prevalence and morbidity associated with CDI after cardiac surgery.
\end{abstract}

\begin{abstract}
Methods: A total of 22,952 patients underwent cardiac surgery at Cleveland Clinic from January 2005 to January 2011. CDI was diagnosed by enzyme immunoassay for toxins and, more recently, polymerase chain reaction (PCR) testing. Hospital outcomes and long-term survival were compared with those of the remaining population in propensity-matched groups.
\end{abstract}

Results: One hundred forty-five patients $(0.63 \%)$ tested positive for CDI at a median of 9 days postoperatively, 135 by enzyme immunoassay and 11 by PCR. Its prevalence more than doubled over the study period. Seventyseven patients $(48 \%)$ were transfers from outside hospitals. Seventy-three patients $(50 \%)$ were exposed preoperatively to antibiotics and $79(56 \%)$ to proton-pump inhibitors. Patients with CDI had more baseline comorbidities, more reoperations, and received more blood products than patients who did not have CDI. Presenting symptoms included diarrhea $(107 ; 75 \%)$, distended abdomen $(48 ; 34 \%)$, and abdominal pain $(27 ; 19 \%)$. All were treated with metronidazole or vancomycin. Sixteen patients $(11 \%)$ died in hospital, including 5 of 10 who developed toxic colitis; 3 of 4 undergoing total colectomy survived. Among matched patients, those with CDI had more septicemia $(P<.0001)$, renal failure $(P=.0002)$, reoperations $(P<.0001)$, prolonged postoperative ventilation $(P<.0001)$, longer hospital stay $(P<.0001)$, and lower 3 -year survival, $52 \%$ versus $64 \%(P=.03)$, than patients who did not have CDI.

Conclusions: Although rare, the prevalence of CDI is increasing, contributing importantly to morbidity and mortality after cardiac surgery. If toxic colitis develops, mortality is high, but colectomy may be lifesaving. (J Thorac Cardiovasc Surg 2014;148:3157-65)

Supplemental material is available online.

Clostridium difficile infection (CDI), caused by a grampositive, spore-forming anaerobic bacillus, is the primary cause of health care-associated diarrhea, ${ }^{1,2}$ and in a study sponsored by the National Institutes of Health, CDI was found to be the third most common infection occurring in the 65 days after cardiac surgery. ${ }^{3}$ It is linked to increasing

\footnotetext{
From the Departments of Thoracic and Cardiovascular Surgery ${ }^{\mathrm{a}}$ and Cardiothoracic Anesthesia, ${ }^{\mathrm{b}}$ Heart and Vascular Institute, Quality and Patient Safety Institute, ${ }^{\mathrm{c}}$ Department of Infectious Disease, ${ }^{\mathrm{d}}$ Medicine Institute, and Department of Quantitative Health Sciences, ${ }^{\mathrm{e}}$ Research Institute, Cleveland Clinic, Cleveland, Ohio. Disclosures: Authors have nothing to disclose with regard to commercial support. Received for publication Dec 16, 2013; revisions received Aug 1, 2014; accepted for publication Aug 6, 2014; available ahead of print Sept 19, 2014.

Address for reprints: Gösta B. Pettersson, MD, PhD, Department of Thoracic and Cardiovascular Surgery, 9500 Euclid Ave, Desk J4-1, Cleveland Clinic, Cleveland, OH 44195 (E-mail: petterg@ccf.org).

$0022-5223 / \$ 36.00$

Copyright (C) 2014 by The American Association for Thoracic Surgery

http://dx.doi.org/10.1016/j.jtcvs.2014.08.017
}

use of antibiotics, yet despite national and global initiatives over the last decade for appropriate antibiotic stewardship, the occurrence of CDI has increased $300 \%$ since $1993 .{ }^{2} \mathrm{Re}$ ports of a more virulent strain of $\mathrm{CDI},{ }^{4-7}$ associated patient morbidity and mortality, and increased resource utilization have further heightened awareness of this complication. ${ }^{2,8,9}$

Our objectives were to (1) determine the prevalence of hospital-acquired CDI after cardiac surgery in our institution, (2) identify patient characteristics and clinical manifestations associated with CDI and its treatment strategies, and (3) assess morbidity and mortality among patients with CDI.

\section{PATIENTS AND METHODS \\ Patients}

From January 2005 to January 2011, 22,952 adults underwent cardiac surgery at Cleveland Clinic. Baseline demographics, comorbidities, and perioperative variables were obtained from the Heart and Vascular Institute's Cardiovascular Information Registry (CVIR). Additional information on perioperative antibiotic use, presenting symptoms, and subsequent treatment of patients diagnosed with CDI was obtained from patient medical records and recorded in a REDCap database. ${ }^{10}$ Cleveland Clinic's Institutional Review Board approved the use of these data for research; patient consent was waived. 


\section{Abbreviations and Acronyms \\ $\mathrm{CDI}=$ Clostridium difficile infection \\ CVIR = Cardiovascular Information Registry \\ EIA = enzyme immunoassay \\ NHLBI $=$ National Heart, Lung, and Blood Institute \\ $\mathrm{PCR}=$ polymerase chain reaction}

\section{Diagnosis of CDI}

CDI was diagnosed by a combination of symptoms and laboratory testing, supplemented in some cases by colonoscopy and laparotomy. These clinically diagnosed cases were entered prospectively into Cleveland Clinic's Infection Prevention surveillance registry. Before the 2nd quarter of 2009, an internal definition of hospital-acquired CDI was used that required a positive enzyme immunoassay (EIA) with onset of diarrhea more than 72 hours after admission or within 72 hours of discharge, or the development of pseudomembranous colitis or toxic megacolon more than 72 hours after admission. Currently, surveillance for hospitalacquired CDI is routinely performed prospectively using standard definitions. ${ }^{11}$ Beginning in October 2010, the hospital's testing platform for CDI changed from an EIA to a polymerase chain reaction (PCR)-based one. In this study, the diagnosis was made by EIA in 135 patients and by PCR in 11. Our yearly incidence density of CDI in the entire hospital from 2006 through 2013 ranged from 6.1 to 11.9 per 10,000 patient-days (Table E1).

\section{End Points}

End points included general complications of cardiac surgery, defined as for the Society of Thoracic Surgeons National Database (see http://www. ctsnet.org/filerptDataSpecifications252_1_ForVendorsPGS.pdf), hospital death, and length of postoperative stay. In addition, specific gastrointestinal symptoms and signs of CDI, including operations performed for CDI complications and their outcome and time-related mortality, were recorded and tabulated for patients with CDI.

Follow-up for death was obtained through the CVIR, supplemented with information from the Social Security Death Master File, queried on October 27, 2011, before its demise in November 2011. ${ }^{12,13}$ A lag time of 6 months resulted in a common closing date of April 27, 2011. Median follow-up for all patients was 2.5 years, with $25 \%$ of patients followed for more than 4.3 years and $10 \%$ more than 5.5 years; 182 patient-years of data were available for analysis in the CDI group. Median follow-up among CDI survivors was 1.4 years, with $25 \%$ followed for more than 2.5 years and $10 \%$ for more than 3.2 years.

\section{Data Analysis}

Data analysis consisted of describing the prevalence of CDI and identifying its incremental risk factors, then using this information as a basis for propensity-matched comparison of hospital outcomes and long-term survival. All analyses were performed using SAS statistical software (SAS version 9.2; SAS Institute, Inc, Cary, NC).

Prevalence of CDI. Cumulative distribution and probability density functions were used to describe temporal data related to CDI. The instantaneous risk of CDI after cardiac surgery was estimated by a multiphase hazard model ${ }^{14}$ in order to determine if risk increased with increasing length of stay.

Risk factors for CDI. Multivariable logistic regression analysis was used to identify patient characteristics associated with CDI. Variable selection from potential risk factors listed in Appendix E1 was by bootstrap aggregation, ${ }^{15,16}$ using 500 bootstrap samples, automated stepwise regression, and a $P$ value retention criterion of .05 . Thereafter, results

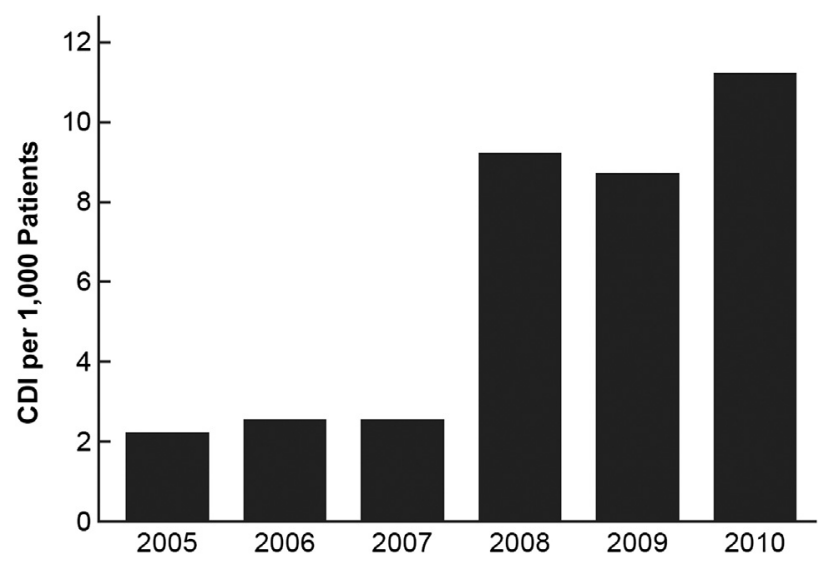

FIGURE 1. Occurrence of in-hospital Clostridium difficile infection $(C D I)$ after cardiac surgery.

were aggregated, and risk factors identified in $50 \%$ of analyses were used to form a parsimonious explanatory model.

This parsimonious model was augmented with preoperative and intraoperative variables representing patient demography, symptoms, cardiac and noncardiac comorbidities, procedures, and support, to form a semisaturated model (see variables so identified in Appendix E1).

Propensity score development. A propensity score was calculated for each patient by solving the saturated model for the probability of being in the CDI group. ${ }^{17-19}$ Then, using only the propensity score, patients who did not have CDI were matched 3 to 1 with patients with CDI using a greedy matching strategy. ${ }^{20}$ All patients with CDI were matched. Figure E1 demonstrates that the resulting propensity-matched CDI and non-CDI groups were well matched.

Comparison of hospital outcomes. Group comparisons were made using the Wilcoxon rank sum test for continuous data and the $\chi^{2}$ test for categorical data (or Fisher exact test when appropriate).

Time-related mortality. Because patients must survive to manifest CDI, death and CDI are of necessity competing risks. Thus, among matched patients, life tables were constructed with all at risk of death initially, with CDI patients censored at the time of diagnosis. Survival of patients with CDI was then estimated from the date of diagnosis. Nonparametric actuarial survival estimates were obtained using the Kaplan-Meier method. A parametric method was used to resolve the number of phases of instantaneous risk of death (hazard function) and to estimate shaping parameters. ${ }^{14}$ (For additional details, see www.lerner.ccf.org/qhs/software/ hazard.)

\section{Presentation of Data}

Continuous variables are summarized as the mean \pm standard deviation and as equivalent 15 th, 50 th (median), and 85 th percentiles when distributions were skewed. Categorical data are summarized by frequencies and percentages. Uncertainty is expressed by confidence limits equivalent to \pm 1 standard error $(68 \%)$.

\section{RESULTS}

\section{Prevalence and Incidence of CDI}

A total of 145 patients $(0.63 \%)$ developed symptomatic CDI after cardiac surgery. Its prevalence increased from 2.2 per 1000 patients in 2005 to 11.2 per 1000 patients in 2010 (Figure 1). Median time from surgery to diagnosis was 9 days (15th and 85 th percentiles, 4 and 22 days, 

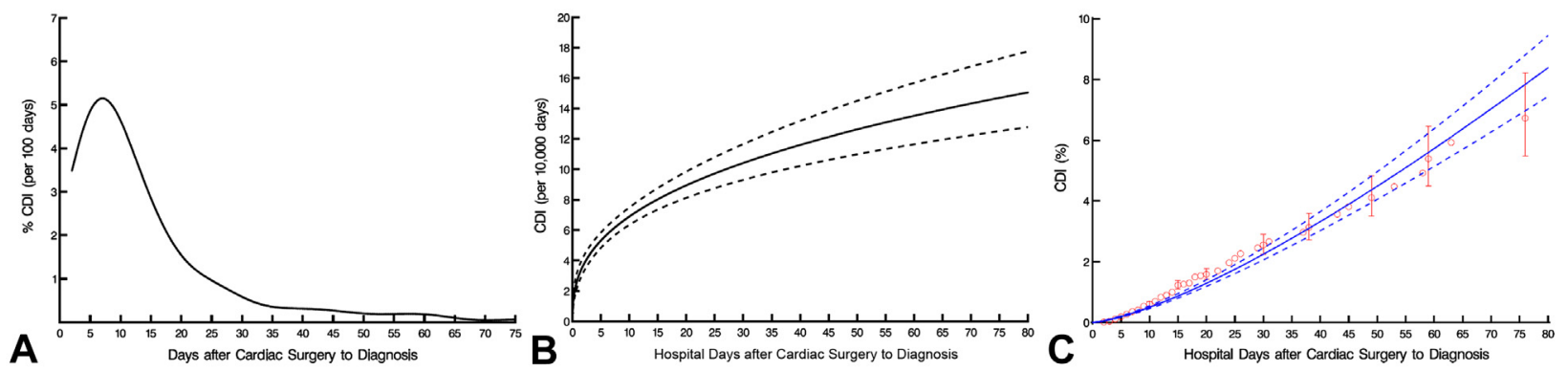

FIGURE 2. Characterization of onset of Clostridium difficile infection (CDI) after cardiac surgery. A, Distribution of the number of days between cardiac surgery and diagnosis. B, Instantaneous in-hospital risk of CDI after cardiac surgery. Solid line is parametric hazard enclosed within a dashed $68 \%$ confidence band equivalent to \pm 1 standard error (SE). C, Occurrence of CDI. Each symbol represents a diagnosis positioned on the vertical axis by the Kaplan-Meier estimator, and vertical bars are confidence limits equivalent to \pm 1 standard error. Solid line is parametric prevalence enclosed within a dashed $68 \%$ confidence band equivalent to $\pm 1 \mathrm{SE}$.

respectively; range, 2-76 days) (Figure 2, A). However, when the competing risk of hospital discharge was accounted for, the incidence rose rapidly in the first few days after surgery, followed by a continued, but lower level, steady increase among patients with prolonged hospitalization (Figure 2, $B$ ). This resulted in an estimated in-hospital prevalence of $0.3 \%$ by 7 days, $2.6 \%$ by 30 days, and $5.5 \%$ by 60 days (Figure 2, C). Median time from peak white blood cell count to CDI diagnosis was 3 days (15th and 85th percentiles, 0 and 15 days, respectively; range, 0-70 days) (Figure E2).

\section{Characterization of Patients With CDI}

Seventy of the 145 patients with CDI (48\%) were transfers from outside hospitals, with a Cleveland Clinic median preoperative stay of 7 days before surgery (Table 1). Fortyfive patients were hospitalized only at Cleveland Clinic before surgery, with a median preoperative stay of 6 days, but 30 had been admitted on the day of surgery. Nine had undergone a colonoscopy for anemia or gastrointestinal symptoms before surgery.

Seventy-three patients had received preoperative antibiotic treatment for reasons varying from routine dental treatment, urinary tract infection, and respiratory infection to endocarditis. Seventy-nine were on proton-pump inhibitors, and 14 were on steroids.

After the heart operation, most patients had a complicated course. A quarter underwent reoperation for bleeding; 131 required inotropic support, and 61 needed a vasopressor. Many required extension of antibiotics beyond the 3 standard doses used for routine prophylaxis, and 96 were started on a new postoperative antibiotic drug for suspected or verified infections, including pneumonia, endocarditis, and wound infections (Table 2). Colonoscopy was required in 4 patients, 2 of whom demonstrated ischemia.

\section{Presenting Symptoms}

The presenting symptoms of CDI were diarrhea in 107 patients, distended abdomen in 48 , and abdominal pain in
27 (see Table 1). Forty-eight patients underwent a computed tomography scan of the abdomen, and $8.5 \%$ had radiologic evidence of colitis, with 6 meeting criteria for pancolitis.

\section{Risk Factors for CDI}

Compared with patients who did not have CDI, those with CDI were older, more symptomatic, and had more comorbidities, such as heart failure, treated diabetes, chronic obstructive pulmonary disease, previous stroke, higher preoperative creatinine level, and lower preoperative hematocrit, and they were more likely to have been hospital transfers (Tables 2 and 3). Surgically, they had longer operative times, longer cardiopulmonary bypass times, and received more blood products.

\section{Treatment}

All 145 patients with CDI were treated with oral or intravenous metronidazole, oral vancomycin, or both (oral metronidazole, 117; intravenous metronidazole, 70; and oral vancomycin, 66; Table 1). Colonoscopy was performed in 9 patients, primarily for bleeding; 3 were reported to have normal results. A clinical picture of toxic colitis developed in 10; 2 were considered inoperable, 1 declined surgery (survived), and 7 underwent emergency laparotomy, with total colectomy in 4 (3 survived), limited resection with stoma in 1 (died), and exploration only in 2 (1 survived; the other died).

\section{Outcomes}

Patients with CDI had substantially more postoperative complications than matched patients without CDI, including strokes, reoperations for bleeding, valve dysfunction, graft occlusion, and renal failure (Table 3). They also had more hospital-acquired infections other than CDI, such as deep sternal wound infections and septicemia. However, among matched patients, hospital outcomes differed primarily with respect to the presence of septicemia, requirement for prolonged ventilation, renal failure, and 
TABLE 1. Patient, procedure, and treatment characteristics of patients before and after developing Clostridium difficile infections $(\mathrm{N}=145)$

\begin{tabular}{|c|c|c|}
\hline Characteristic & $\begin{array}{c}\text { No. of } \\
\text { patients } \\
\text { with data } \\
\text { available }\end{array}$ & No. $(\%)$ \\
\hline \multicolumn{3}{|l|}{ Preoperative } \\
\hline History of CDI & 145 & $5(3.4)$ \\
\hline Hospital transfer & 145 & $67(46)$ \\
\hline In ICU before surgery & 145 & $48(33)$ \\
\hline Preoperative antibiotic & 145 & $72(50)$ \\
\hline Reason for antibiotic & 72 & \\
\hline Urinary tract infection & & $16(22)$ \\
\hline Respiratory infection & & $16(22)$ \\
\hline Endocarditis & & $13(18)$ \\
\hline Dental treatment & & $8(11)$ \\
\hline Other infection & & $19(26)$ \\
\hline Proton-pump inhibitor & 145 & $81(56)$ \\
\hline Steroids & 145 & $15(10)$ \\
\hline Immunosuppression (other) & 145 & $7(4.8)$ \\
\hline Bowel preparation within $30 \mathrm{~d}$ of surgery & 145 & $15(10)$ \\
\hline Nasogastric tube & 145 & $3(2.0)$ \\
\hline Previous abdominal surgery & 145 & $57(39)$ \\
\hline History of IBD & 145 & $8(5.5)$ \\
\hline Colonoscopy & 145 & $9(6.2)$ \\
\hline Normal & & $3(33)$ \\
\hline Diverticulosis & & $4(44)$ \\
\hline Bleeding rectal ulcer & & $1(11)$ \\
\hline Suspected ischemia & & $1(11)$ \\
\hline \multicolumn{3}{|l|}{ Peri- and postoperative (before CDI diagnosis) } \\
\hline Prophylactic antibiotic extended after 3 doses & 145 & $32(22)$ \\
\hline New postoperative antibiotic & 145 & $96(66)$ \\
\hline Reason for antibiotics & 96 & \\
\hline High white blood cell count & & $71(74)$ \\
\hline Fever & & $36(38)$ \\
\hline Pneumonia & & $32(33)$ \\
\hline Reoperation for bleeding & & $23(24)$ \\
\hline Wound infection & & $14(15)$ \\
\hline Endocarditis & & $13(13)$ \\
\hline Other & & $40(42)$ \\
\hline Inotrope & 145 & $132(91)$ \\
\hline Vasopressor & 145 & $62(43)$ \\
\hline Tracheostomy & 145 & $41(28)$ \\
\hline Bleeding/reexploration & 145 & $35(24)$ \\
\hline Colonoscopy & 145 & $4(2.8)$ \\
\hline Ischemia & & $2(50)$ \\
\hline Polyp & & $1(25)$ \\
\hline Normal & & $1(25)$ \\
\hline \multicolumn{3}{|l|}{ Symptoms and diagnosis of CDI } \\
\hline Diarrhea & 145 & $109(75)$ \\
\hline Abdominal distension & 145 & $49(34)$ \\
\hline Fever & 145 & $47(32)$ \\
\hline Abdominal pain & 145 & $27(19)$ \\
\hline CT scan & 145 & $48(33)$ \\
\hline Colitis & & $14(29)$ \\
\hline Pancolitis & & $6(13)$ \\
\hline
\end{tabular}

TABLE 1. Continued

\begin{tabular}{lcc}
\hline \multicolumn{1}{c}{ Characteristic } & $\begin{array}{c}\text { No. of } \\
\text { patients } \\
\text { with data } \\
\text { available }\end{array}$ & No. (\%) \\
\hline Free air & & $0(0)$ \\
Distended bowel & & $13(27)$ \\
Normal & & $15(31)$ \\
Management of CDI & 145 & $145(100)$ \\
Antibiotics* & & $117(81)$ \\
Oral flagyl & & $70(48)$ \\
Intravenous flagyl & & $66(46)$ \\
Oral vancomycin & 145 & $9(6.2)$ \\
Colonoscopy & & $2(22)$ \\
Colitis & & $1(11)$ \\
Rectal ulcer & & $1(11)$ \\
Diverticula and AVMs & $2(22)$ \\
AVMs & & $3(44)$ \\
Normal or nonspecific & 145 & $7(4.8)$ \\
Surgery/laparotomy & & $1(14)$ \\
Limited bowel resection with stoma & $4(57)$ \\
Colectomy & & $2(29)$ \\
Exploration & & $3(3.4)$ \\
Microscopy (operative specimen) & & $1(20)$ \\
Pseudomembranous colitis & & $1(20)$ \\
Ischemic colitis & & \\
Cytomegalovirus & & \\
\hline CDI, Clostridium difficile infection; ICU, intensive care unit; IBD, inflammatory \\
bowel disease; $C T$, computed tomography; AVMs, arteriovenous malformations. \\
$*$ Not mutually exclusive.
\end{tabular}

reoperation for bleeding. Resource utilization was high, as reflected in longer duration of postoperative ventilatory support, and longer stays in the intensive care unit and in hospital (Table 4). In-hospital mortality was higher among patients with CDI (11\%), particularly if toxic colitis developed $(50 \%$, see earlier), but was similar among matched patients.

Survival after diagnosis of CDI at 30 days, 6 months, and 1,2 , and 3 years was $88 \%, 70 \%, 63 \%, 59 \%$, and $52 \%$, respectively (Figure E3). Survival among matched patients without CDI at the same intervals was $90 \%, 82 \%, 77 \%$, $70 \%$, and $64 \%(P=.03$ by time-varying covariable analysis; Figure 3), with a prolonged higher risk of death for at least 6 months.

\section{DISCUSSION}

Prevalence and Incidence of CDI

We report an increasing prevalence of CDI that parallels national and global trends. ${ }^{2}$ In part, this may reflect more sensitive testing, but the increase in prevalence was evident a year before PCR techniques supplanted EIA, and the increase was greater for patients undergoing cardiac surgery than for the hospital as a whole (Table E1).

Although time to diagnosis peaked at 6 to 7 days after surgery then decreased sharply, this does not take into 
TABLE 2. Patient and procedure characteristics: before matching and matched groups

\begin{tabular}{|c|c|c|c|c|c|c|c|c|}
\hline \multirow[b]{2}{*}{ Variable } & \multicolumn{3}{|c|}{ No CDI: before matching $(n=22,807)$} & \multicolumn{2}{|c|}{$\operatorname{CDI}(n=145)$} & \multicolumn{3}{|c|}{ No CDI: matched $(n=435)$} \\
\hline & $\begin{array}{c}\text { No. of } \\
\text { patients } \\
\text { with data } \\
\text { available }\end{array}$ & $\begin{array}{l}\text { No. }(\%) \text { or } \\
\text { mean } \pm \text { SD }\end{array}$ & $P^{*}$ & $\begin{array}{c}\text { No. of } \\
\text { patients } \\
\text { with data } \\
\text { available } \\
\end{array}$ & $\begin{array}{l}\text { No. }(\%) \text { or } \\
\text { mean } \pm \text { SD }\end{array}$ & $\boldsymbol{P} \dagger$ & $\begin{array}{c}\begin{array}{c}\text { No. of } \\
\text { patients }\end{array} \\
\text { with data } \\
\text { available } \\
\end{array}$ & $\begin{array}{l}\text { No. }(\%) \text { or } \\
\text { mean } \pm \text { SD }\end{array}$ \\
\hline \multicolumn{9}{|l|}{ Demographics } \\
\hline Age (y) & 22,807 & $63 \pm 14$ & .001 & 145 & $67 \pm 14$ & .7 & 435 & $67 \pm 14$ \\
\hline Female & 22,807 & $7818(34)$ & .15 & 145 & $58(40)$ & $>.9$ & 435 & $174(40)$ \\
\hline Race & 22,634 & & & 145 & & & 434 & \\
\hline White & & $20,145(89)$ & .18 & & $124(86)$ & .6 & & $379(87)$ \\
\hline Black & & $1425(6.3)$ & .10 & & $14(9.7)$ & 6 & & $36(8.3)$ \\
\hline Other & & $1064(4.7)$ & $>.9$ & & $7(4.8)$ & .8 & & $19(4.4)$ \\
\hline Body surface area $\left(\mathrm{m}^{2}\right)$ & 22,241 & $2.02 \pm 0.28$ & .4 & 144 & $2.01 \pm 0.29$ & .3 & 425 & $1.98 \pm 0.28$ \\
\hline Hospital transfer & 22,671 & $4788(21)$ & $<.0001$ & 145 & $70(48)$ & .8 & 435 & $206(47)$ \\
\hline \multicolumn{9}{|l|}{ Symptoms } \\
\hline NYHA functional class & 22,588 & & $<.0001$ & 145 & & .8 & 434 & \\
\hline I & & $6141(27)$ & & & $21(14)$ & & & $55(13)$ \\
\hline II & & $9555(42)$ & & & $47(32)$ & & & $156(36)$ \\
\hline III & & $5573(25)$ & & & $54(37)$ & & & $147(34)$ \\
\hline IV & & $1319(5.8)$ & & & $23(16)$ & & & $78(18)$ \\
\hline Emergency surgery & 21,882 & & .8 & 145 & $5(3.4)$ & .4 & 421 & $22(5.2)$ \\
\hline \multicolumn{9}{|l|}{ Cardiac comorbidity } \\
\hline $\operatorname{LVEF}(\%)$ & 14,323 & $51 \pm 13$ & .002 & 57 & $43 \pm 17$ & .5 & 192 & $45 \pm 16$ \\
\hline Myocardial infarction & 22,807 & $5829(26)$ & .01 & 145 & $50(34)$ & .4 & 435 & $134(31)$ \\
\hline Heart failure & 22,787 & $6081(27)$ & $<.0001$ & 145 & $67(46)$ & .8 & 435 & $195(45)$ \\
\hline Endocarditis & 22,788 & $1250(5.5)$ & .3 & 145 & $11(7.6)$ & .7 & 435 & $37(8.5)$ \\
\hline Previous cardiac surgery & 22,802 & $6365(28)$ & .3 & 145 & $46(32)$ & .7 & 435 & $145(33)$ \\
\hline \multicolumn{9}{|l|}{ Noncardiac comorbidity } \\
\hline Hypertension & 22,788 & $15,974(70)$ & .7 & 145 & $104(72)$ & .8 & 435 & $307(71)$ \\
\hline Stroke & 22,807 & $2161(9.5)$ & .004 & 145 & $24(17)$ & .8 & 435 & $69(16)$ \\
\hline Pharmacologically treated diabetes & 22,566 & $4530(20)$ & .008 & 141 & $41(29)$ & .5 & 434 & $114(26)$ \\
\hline PAD & 22,807 & $2220(9.7)$ & .10 & 145 & $20(14)$ & .9 & 435 & $62(14)$ \\
\hline COPD & 22,787 & $3445(15)$ & $<.0001$ & 145 & $48(33)$ & .9 & 435 & $147(34)$ \\
\hline Smoking & 22,701 & $12,181(54)$ & .11 & 144 & $87(60)$ & 6 & 434 & $272(63)$ \\
\hline Creatinine (mg/dL) & 22,786 & $1.2 \pm 0.90$ & $<.0001$ & 145 & $1.6 \pm 1.3$ & .5 & 434 & $1.5 \pm 1.3$ \\
\hline Renal dialysis & 22,787 & $387(1.7)$ & $<.0001$ & 145 & $9(6.2)$ & .15 & 435 & $15(3.4)$ \\
\hline Hematocrit $(\%)$ & 22,801 & $38 \pm 6.3$ & $<.0001$ & 145 & $35 \pm 6.0$ & $>.9$ & 434 & $35 \pm 6.4$ \\
\hline \multicolumn{9}{|l|}{ Procedure } \\
\hline Isolated CABG & 22,807 & $3940(17)$ & .7 & 145 & $23(16)$ & .8 & 435 & $72(17)$ \\
\hline Isolated valve & 22,807 & $6918(30)$ & .07 & 145 & $34(23)$ & .4 & 435 & $88(20)$ \\
\hline Combined CABG/valve & 22,807 & $3283(14)$ & .002 & 145 & $34(23)$ & 6 & 435 & $92(21)$ \\
\hline Aortic root, ascending aorta & 22,807 & $3990(17)$ & .6 & 145 & $23(16)$ & .2 & 435 & $89(20)$ \\
\hline Other cardiac & 22,807 & $4676(21)$ & .8 & 145 & $31(21)$ & .9 & 435 & $94(22)$ \\
\hline Surgical invasiveness & 22,042 & & & 145 & & & 419 & \\
\hline Full incision & & $17,818(81)$ & $<.0001$ & & $136(94)$ & 6 & & $398(95)$ \\
\hline Minimal & & $3130(14)$ & .006 & & $9(6.2)$ & .14 & & $14(3.3)$ \\
\hline Percutaneous/ports & & $1094(4.8)$ & .007 & & $0(0)$ & .19 & & $7(1.7)$ \\
\hline CPB time (min) & 22,807 & $95 \pm 55$ & $<.0001$ & 145 & $128 \pm 58$ & .16 & 435 & $120 \pm 65$ \\
\hline Myocardial ischemic time (min) & 22,807 & $69 \pm 42$ & .0004 & 145 & $83 \pm 53$ & .19 & 435 & $77 \pm 50$ \\
\hline Intra-/postoperative IABP & 22,807 & $566(2.5)$ & $<.0001$ & 145 & $17(12)$ & .3 & 435 & $39(9.0)$ \\
\hline Blood product use & 22,807 & $11,456(50)$ & & 145 & $134(92)$ & & 435 & $386(89)$ \\
\hline $\mathrm{RBC}$ transfusion & & $10,491(46)$ & $<.0001$ & & $128(88)$ & .18 & & $364(84)$ \\
\hline FFP & & $4869(21)$ & $<.0001$ & & $84(58)$ & .03 & & $206(47)$ \\
\hline
\end{tabular}


TABLE 3. Incremental risk factors for Clostridium difficile infection

\begin{tabular}{|c|c|c|c|c|}
\hline Risk factor & Estimate \pm SE & Odds ratio (68\% CLs) & $\boldsymbol{P}$ & Reliability $(\%) *$ \\
\hline \multicolumn{5}{|l|}{ Demographics } \\
\hline Older age $\dagger$ & $0.21 \pm 0.092$ & See Figure E4, $A$ & .02 & 50 \\
\hline Hospital transfer & $0.44 \pm 0.179$ & $1.5(1.3,1.8)$ & .006 & 71 \\
\hline \multicolumn{5}{|l|}{ Comorbidities } \\
\hline Lower HDL & $-0.021 \pm 0.0065$ & See Figure E4, $B$ & .001 & 67 \\
\hline Increased creatinine $\ddagger$ & $0.36 \pm 0.158$ & See Figure E4, $C$ & .02 & 51 \\
\hline COPD & $0.71 \pm 0.182$ & $2.0(1.7,2.4)$ & $<.0001$ & 90 \\
\hline Hypertension (less risk) & $-0.45 \pm 0.196$ & $0.64(0.52,0.77)$ & .02 & 58 \\
\hline \multicolumn{5}{|l|}{ Procedural } \\
\hline Insertion of temporary mechanical assist device & $0.94 \pm 0.23$ & $2.9(2.4,3.7)$ & $<.0001$ & 93 \\
\hline Blood use intra- or postoperatively & $1.3 \pm 0.37$ & $3.9(2.7,5.6)$ & .0003 & 80 \\
\hline Intra- and postoperative cardiac $\mathrm{RBC}$ transfusion units $\S$ & $0.44 \pm 0.110$ & See Figure E4, $D$ & $<.0001$ & 59 \\
\hline More recent date of operation & $0.32 \pm 0.057$ & See Figure E4, $E$ & $<.0001$ & 99 \\
\hline Intercept & $-7.83 \pm 0.56$ & & $<.0001$ & - \\
\hline
\end{tabular}

C-statistic $=0.85 . S E$, Standard error; $C L$, confidence limits; $H D L$, high-density lipoprotein cholesterol; $C O P D$, chronic obstructive pulmonary disease; $R B C$, red blood cell. *Percent of times factor appeared in 500 bootstrap models. $\dagger \operatorname{Exp}($ age/50), exponential transformation. $\ddagger \log ($ creatinine), logarithmic transformation. $\S \mathrm{Log}(\mathrm{RBC}$ units), logarithmic transformation.

account the rapidly diminishing number of patients not yet discharged after 7 days. The hazard function accounts for this and demonstrates that risk of CDI continues to increase the longer a patient is hospitalized. As a result, cumulative incidence of CDI increases exponentially to more than 5\% at 60 days. Although an inference might be that continued hospital exposure is the cause of this increasing incidence, we caution the reader that we do not know whether patients who did not have CDI may have been diagnosed with CDI after hospital discharge. The National Heart, Lung, and Blood Institute (NHLBI)-funded Cardiothoracic Surgery Clinical Trials Network infection study, which extended to 60 days beyond hospital discharge, showed that nearly half the infections occurred after discharge. Thus, the general pattern of incidence of infection, including CDI, is unclear because these late infections have not previously been studied.

The epidemiology of CDI has changed over time, with reports of a previously uncommon and more virulent strain, BI/NAP1/027, increasingly associated with disease outbreaks. ${ }^{4-7}$ This strain is characterized by a 16 - to 23 -fold increase in production of toxins A and B. ${ }^{7}$ Hypervirulence is manifested clinically by a high fever, unstable hemodynamics, and the development of toxic megacolon. ${ }^{8}$ Some have hypothesized that widespread use of fluoroquinolone antibiotics may have selectively allowed for proliferation of this strain. ${ }^{11}$ We do not know how many of our patients with CDI had this strain.

TABLE 4. Outcomes before matching with and without CDI, and matched group

\begin{tabular}{|c|c|c|c|c|c|}
\hline \multirow[b]{2}{*}{ Variable } & \multicolumn{2}{|c|}{ No CDI: before matching $(n=22,807)$} & \multirow{2}{*}{$\frac{\operatorname{CDI}(n=145)}{\text { No. }(\%) \text { or mean } \pm \text { SD }}$} & \multicolumn{2}{|c|}{ No CDI: matched $(n=435)$} \\
\hline & No. $(\%)$ or mean \pm SD & $\boldsymbol{P}^{*}$ & & $\boldsymbol{P} \dagger$ & No. $(\%)$ or mean \pm SD \\
\hline \multicolumn{6}{|l|}{ Complications } \\
\hline Septicemia & $562(2.5)$ & $<.0001$ & $19(13)$ & $<.0001$ & $15(3.4)$ \\
\hline Deep sternal wound infection & $148(0.70)$ & .07 & $3(2.1)$ & .4 & $4(0.92)$ \\
\hline Hospital death & $820(3.6)$ & $<.0001$ & $16(11)$ & .8 & $44(10)$ \\
\hline Permanent stroke & $427(1.9)$ & .001 & $8(5.5)$ & .3 & $15(3.4)$ \\
\hline Perioperative MI & $66(0.30)$ & .5 & $0(0)$ & $>.9$ & $2(0.46)$ \\
\hline Atrial fibrillation & $5509(24)$ & .0001 & $55(38)$ & .07 & $130(30)$ \\
\hline Ventilation $>24 \mathrm{~h}$ & 4299 (19) & $<.0001$ & $96(66)$ & $<.0001$ & $194(45)$ \\
\hline Renal failure requiring dialysis & $615(2.7)$ & $<.0001$ & $24(17)$ & .002 & $33(7.6)$ \\
\hline Reoperation for bleeding & $1100(4.8)$ & $<.0001$ & $20(14)$ & .03 & $34(7.8)$ \\
\hline \multicolumn{6}{|l|}{ Lengths of stay $\ddagger$} \\
\hline ICU $(\mathrm{h}) \S$ & $40(22,126)$ & $<.0001$ & $264(69,801)$ & $<.0001$ & $90(24,281)$ \\
\hline Postoperative (d) $\S$ & $7.1(4.9,15)$ & $<.0001$ & $21(10,49.5)$ & $<.0001$ & $11(6.1,26)$ \\
\hline Hospital (d) $\S$ & $9.0(5.3,21)$ & $<.0001$ & $27(13,59)$ & $<.0001$ & $16(8.0,36)$ \\
\hline
\end{tabular}

CDI, Clostridium difficile infection; $S D$, standard deviation; $M I$, myocardial infarction; $I C U$, intensive care unit. *Unmatched versus CDI. $\dagger$ Matched versus CDI. $\ddagger$ Median (15th, 85th percentiles). §No CDI: before matching group. Data available for 22,660 patients. 


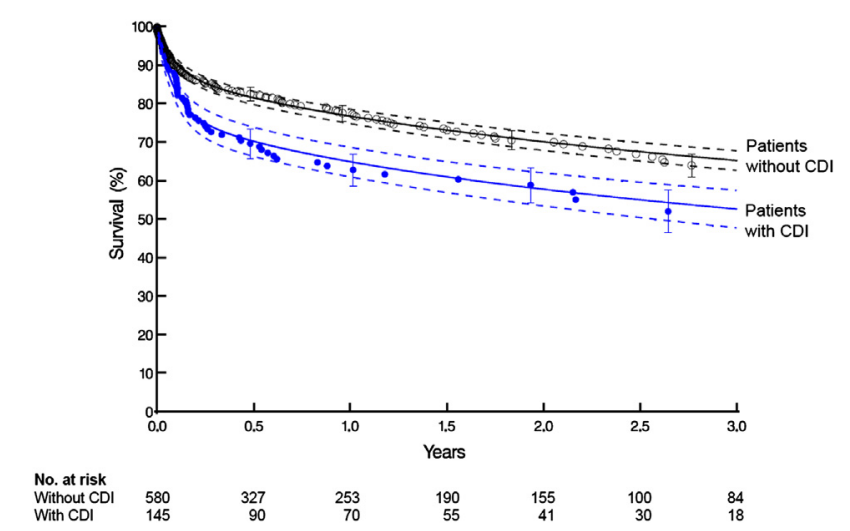

FIGURE 3. Survival of propensity-matched patients stratified by whether or not the patient had a Clostridium difficile infection $(C D I)$. Time zero for the upper curve is the date of surgery, with patients censored at occurrence of CDI (competing-risks analysis). Time zero for the lower curve is the time of CDI diagnosis. Each symbol represents a death, vertical bars are $68 \%$ confidence limits equivalent to \pm 1 standard error, and solid lines are parametric estimates enclosed within a $68 \%$ confidence band.

\section{Characteristics of Patients With CDI and Risk Factors for CDI}

Our patients with CDI had several previously welldescribed risk factors, including previous exposure to antibiotics, ${ }^{8,21,22}$ preoperative hospitalization, and longer duration of hospitalization. ${ }^{23,24}$ Half had been treated with broadspectrum antibiotics before the CDI diagnosis. ${ }^{21,22,25,26}$ More than half were on a proton-pump inhibitor, which is linked to increased occurrence of $\mathrm{CDI}^{27-29}$ Increased gastric $\mathrm{pH}$ allows survival of CDI spores. ${ }^{28,29}$

Other known risk factors illustrated by our study included older age $1,2,8,21,22,26$ and previous colonoscopy or gastrointestinal surgery ${ }^{1,21,28}$; some patients were immunocompromised. $^{1,9,28}$ Patients with CDI also had a high comorbidity burden, which is associated with greater susceptibility to CDI. ${ }^{30,31}$

Only 5 of our patients had a history of CDI, and none had been diagnosed as carriers. ${ }^{32}$ This may suggest that symptomless colonization with $C$ difficile is protective and associated with a reduced risk for $C$ difficile-associated diarrhea. Others have noted that an alteration in normal fecal flora, followed by colonization with $C$ difficile and subsequent release of toxins, are necessary steps in the development of $C$ difficile diarrhea. ${ }^{33}$

Although a limitation of our study is that previous antibiotic use, previous gastrointestinal surgery, immunocompromise, and proton-pump inhibitor use were known only for CDI patients, surrogates for this, such as hospital transfer status, chronic pulmonary disease, and some comorbidities known for all patients, were associated with the occurrence of CDI. However, during multivariable analysis, it was evident that surgical factors such as blood product use and the need to insert a temporary circulatory assist device were powerful risk factors. Both cardiopulmonary bypass and blood product use are associated with alterations in immunology and elaboration of cytokines. ${ }^{34}$ Both have the potential to raise levels of free iron, which is associated with infection. ${ }^{35}$ We have investigated these associations in detail at this institution, particularly red blood cell transfusion, ${ }^{36}$ and our findings have been corroborated by the NHLBI-funded Cardiothoracic Surgery Clinical Trials Network postcardiac surgery infection study. ${ }^{3}$ The mechanism remains elusive, but it further emphasizes the value of blood conservation and protocols to reduce intraoperative bleeding and the use of blood products. The study also highlights the vulnerability of patients who require temporary circulatory support after cardiac surgery, with the potential for gut ischemia from low cardiac output.

\section{Presentation and Diagnosis}

More than $70 \%$ of our patients presented with diarrhea, as well as abdominal pain, distension, and leukocytosis. Cytotoxins produced by $C$ difficile lead to symptoms, typically within 48 to 72 hours of infection. ${ }^{21}$

Laboratory diagnosis of CDI involved testing for stool toxins and, less commonly, stool cultures. Our change in the testing platform from EIA to PCR was based on the Hookman and Barkin ${ }^{21}$ report of laboratory testing for CDI: EIA, 73\% sensitivity, 98\% specificity; real-time PCR, 93\% sensitivity, 97\% specificity; cell culture assay, $77 \%$ sensitivity, $97 \%$ specificity; and anaerobic culture for toxigenic $C$ difficile strains, $100 \%$ sensitivity, $96 \%$ specificity. Although stool cultures are sensitive tests, they are associated with long turnaround times compared with the relatively rapid turnaround times for PCR testing. ${ }^{37}$ If colonoscopy is performed, it may demonstrate colonic inflammation and, if CDI is advanced, may show the presence of pseudomembranes, diagnostic for CDI. ${ }^{38}$ The fact that only one third of the patients had a computed tomography scan and only 9 had a colonoscopy, and one third of these examinations were read as normal, strongly suggests that the abdominal presentation of CDI was relatively benign in most patients.

\section{Treatment}

All our patients were treated with antibiotic therapy (vancomycin, metronidazole, or both). Patients with clinical symptoms of toxic colitis were offered surgery if operable. Three of 4 patients undergoing colectomy survived.

We have not tried treatment with monoclonal antibodies against $C$ difficile toxins, which has been reported to be successful. ${ }^{39}$ Nor have we used nitazoxanide and rifaximin, fidaxomicin, probiotics, or anionic polymers, as suggested by others. ${ }^{8,38}$ Antibiotic treatment does not reestablish normal flora in the bowel; hence, some advocate fecal bacteriotherapy for patients with chronic relapsing CDI who fail conventional treatment. ${ }^{9,40-42}$ 


\section{Outcomes}

CDI was associated with increased hospital morbidity, mortality, and resource utilization, and prolonged high risk of death for least 6 months after surgery. Complications related to CDI led in part to this increased use of resources via the need for additional surgical procedures, pharmacotherapy, and increased length of stay. However, in most of our cases, the CDI manifestations were benign and the CDI was just another marker of the severity of comorbidities and patient and operative complexity. ${ }^{43}$ In other general hospital settings, higher mortality, longer lengths of stay, and higher costs have been documented. ${ }^{44}$

\section{Clinical Implications}

The increasing prevalence of CDI after heart surgery is concerning and costly, and more effective efforts to limit its spread are needed. Our study has shown that the complex and complicated patients are most vulnerable.

The mode of CDI transmission is via the fecal-oral route of $C$ difficile spores; hence, vigilance regarding environmental reservoirs, including asymptomatic $C$ difficile carriers, is warranted. $C$ difficile spores are resilient and can survive on surfaces for months despite standard sterilization treatments. $^{21,38}$ We believe that a high level of suspicion plus early diagnosis and treatment can reduce the deleterious consequences.

\section{Strengths and Limitations}

This study was conducted in a quaternary care setting with high-acuity patients undergoing cardiac surgery, $20 \%$ of whom were transferred from other hospitals. Thus, our findings may not be generalizable to other settings. One strength of our study is the detailed information available case by case for patients with CDI, which cannot be achieved with purely administrative data. However, as noted earlier, many traditional risk factors for CDI were not available for the patients without CDI. Toward the end of the study period, diagnostic testing evolved, and it is not clear how this affected the detection of CDI. We also recognize that CDI cannot be randomized, and the use of propensity score methods for so-called natural experiments such as this may be considered controversial by some. We believe, however, that risk adjustment is superior to multivariable analysis of outcomes. ${ }^{18}$

\section{CONCLUSIONS}

CDI is an important contributor to morbidity and mortality after cardiac surgery, and the risk of CDI increases during prolonged hospitalization. Although risk factors such as prolonged hospitalization, antibiotics, proton-pump inhibitors, comorbidities, and high-risk operations were common among patients with CDI, the strongest risk factors in this setting were perioperative ones, including blood product use and the need for temporary circulatory support. If toxic colitis develops, mortality is high, but colectomy may be lifesaving.

\section{References}

1. Kim JH, Muder RR. Clostridium difficile enteritis: a review and pooled analysis of the cases. Anaerobe. 2011;17:52-5.

2. Lucado J, Gould C, Elixhauser A. Clostridium difficile infections (CDI) in hospital stays, 2009. HCUP statistical brief \#124. Rockville, MD: Agency for Healthcare Research and Quality; January 2012. Available at: www.ahrq.gov/ news.

3. Horvath KA, Acker MA, Chang H, Bagiella E, Smith PK, Iribarne A, et al. Blood transfusion and infection after cardiac surgery. Ann Thorac Surg. 2013;95: 2194-201.

4. O'Connor JR, Johnson S, Gerding DN. Clostridium difficile infection caused by the epidemic BI/NAP1/027 strain. Gastroenterology. 2009;136:1913-24.

5. McDonald LC, Killgore GE, Thompson A, Owens RC Jr, Kazakova SV, Sambol SP, et al. An epidemic, toxin gene-variant strain of Clostridium difficile. N Engl J Med. 2005;353:2433-41.

6. Walker AS, Eyre DW, Wyllie DH, Dingle KE, Harding RM, O'Connor L, et al. Characterisation of Clostridium difficile hospital ward-based transmission using extensive epidemiological data and molecular typing. PLoS Med. 2012;9: e1001172.

7. Pant C, Sferra TJ, Deshpande A, Minocha A. Clinical approach to severe Clostridium difficile infection: update for the hospital practitioner. Eur J Intern Med. 2011;22:561-8.

8. Gould CV, McDonald LC. Bench-to-bedside review: Clostridium difficile colitis. Crit Care. 2008;12:203.

9. Flagg A, Koch CG, Schiltz N, Pillai C, Gordon SM, Pettersson GB, et al. Analysis of Clostridium difficile infections after cardiac surgery: epidemiologic and economic implications from national data. J Thorac Cardiovasc Surg. April 13, 2014 [Epub ahead of print].

10. Harris PA, Taylor R, Thielke R, Payne J, Gonzalez N, Conde JG. Research electronic data capture (REDCap)-a metadata-driven methodology and workflow process for providing translational research informatics support. J Biomed Inform. 2009; 42:377-81.

11. McDonald LC, Coignard B, Dubberke E, Song X, Horan T, Kutty PK. Recommendations for surveillance of Clostridium difficile-associated disease. Infect Control Hosp Epidemiol. 2007;28:140-5.

12. Boyle CA, Decoufle P. National sources of vital status information: extent of coverage and possible selectivity in reporting. Am J Epidemiol. 1990;131:160-8.

13. Newman TB, Brown AN. Use of commercial record linkage software and vital statistics to identify patient deaths. J Am Med Inform Assoc. 1997;4:233-7.

14. Blackstone EH, Naftel DC, Turner ME Jr. The decomposition of time-varying hazard into phases, each incorporating a separate stream of concomitant information. J Am Stat Assoc. 1986;81:615-24.

15. Breiman L. Bagging predictors. Machine Learning. 1996;24:123-40.

16. Sauerbrei W, Schumacher M. A bootstrap resampling procedure for model building: application to the Cox regression model. Stat Med. 1992;11:2093-109.

17. Rosenbaum PR, Rubin DB. The central role of the propensity score in observational studies for causal effects. Biometrika. 1983;70:41-55.

18. Rubin DB. The design versus the analysis of observational studies for causal effects: parallels with the design of randomized trials. Stat Med. 2007;26:20-36.

19. Blackstone EH. Comparing apples and oranges. J Thorac Cardiovasc Surg. 2002; 123:8-15.

20. Bergstralh EJ, Konsanke JL. Computerized matching of cases to controls. Technical report no. 56. Rochester, Minn: Department of Health Science Research, Mayo Clinic; 1995.

21. Hookman P, Barkin JS. Clostridium difficile associated infection, diarrhea and colitis. World J Gastroenterol. 2009;15:1554-80.

22. Diggs NG, Surawicz CM. Evolving concepts in Clostridium difficile colitis. Curr Gastroenterol Rep. 2009; 11:400-5.

23. Bartlett JG, Gerding DN. Clinical recognition and diagnosis of Clostridium diffcile infection. Clin Infect Dis. 2008;46(Suppl 1):S12-8.

24. Pepin J, Saheb N, Coulombe MA, Alary ME, Corriveau MP, Authier S, et al. Emergence of fluoroquinolones as the predominant risk factor for Clostridium difficile-associated diarrhea: a cohort study during an epidemic in Quebec. Clin Infect Dis. 2005;41:1254-60.

25. Johnson S, Gerding DN. Clostridium difficile-associated diarrhea. Clin Infect Dis. 1998;26:1027-34; quiz 35-6. 
26. Crabtree T, Aitchison D, Meyers BF, Tymkew H, Smith JR, Guthrie TJ, et al. Clostridium difficile in cardiac surgery: risk factors and impact on postoperative outcome. Ann Thorac Surg. 2007;83:1396-402.

27. Dial S, Delaney JA, Barkun AN, Suissa S. Use of gastric acid-suppressive agents and the risk of community-acquired Clostridium difficile-associated disease. JAMA. 2005;294:2989-95.

28. Cunningham R, Dial S. Is over-use of proton pump inhibitors fuelling the current epidemic of Clostridium difficile-associated diarrhoea? J Hosp Infect. 2008;70: 1-6.

29. Aseeri M, Schroeder T, Kramer J, Zackula R. Gastric acid suppression by proton pump inhibitors as a risk factor for Clostridium difficile-associated diarrhea in hospitalized patients. Am J Gastroenterol. 2008;103:2308-13.

30. Hardt C, Berns T, Treder W, Dumoulin FL. Univariate and multivariate analysis of risk factors for severe Clostridium difficile-associated diarrhoea: importance of co-morbidity and serum C-reactive protein. World J Gastroenterol. 2008;14: 4338-41.

31. Rodrigues MA, Brady RR, Rodrigues J, Graham C, Gibb AP. Clostridium diffcile infection in general surgery patients; identification of high-risk populations. Int J Surg. 2010;8:368-72.

32. Shim JK, Johnson S, Samore MH, Bliss DZ, Gerding DN. Primary symptomless colonisation by Clostridium difficile and decreased risk of subsequent diarrhoea. Lancet. 1998;351:633-6.

33. Poutanen SM, Simor AE. Clostridium difficile-associated diarrhea in adults. Can Med Assoc J. 2004;171:51-8.

34. Fransen E, Maessen J, Dentener M, Senden N, Buurman W. Impact of blood transfusions on inflammatory mediator release in patients undergoing cardiac surgery. Chest. 1999;116:1233-9.

35. Hod EA, Brittenham GM, Billote GB, Francis RO, Ginzburg YZ, Hendrickson JE, et al. Transfusion of human volunteers with older, stored red blood cells produces extravascular hemolysis and circulating non-transferrinbound iron. Blood. 2011;118:6675-82.

36. Koch CG, Li L, Duncan AI, Mihaljevic T, Cosgrove DM, Loop FD, et al. Morbidity and mortality risk associated with red blood cell and blood-component transfusion in isolated coronary artery bypass grafting. Crit Care Med. 2006;34:1608-16.

37. Cohen SH, Gerding DN, Johnson S, Kelly CP, Loo VG, McDonald LC, et al Clinical practice guidelines for Clostridium difficile infection in adults: 2010 update by the Society for Healthcare Epidemiology of America (SHEA) and the Infectious Diseases Society of America (IDSA). Infect Control Hosp Epidemiol. 2010;31:431-55.

38. Heinlen L, Ballard JD. Clostridium difficile infection. Am J Med Sci. 2010;340: 247-52.

39. Lowy I, Molrine DC, Leav BA, Blair BM, Baxter R, Gerding DN, et al. Treatment with monoclonal antibodies against Clostridium difficile toxins. $N$ Engl $J$ Med. 2010:362:197-205.

40. Bakken JS. Fecal bacteriotherapy for recurrent Clostridium difficile infection Anaerobe. 2009;15:285-9.

41. Yoon SS, Brandt LJ. Treatment of refractory/recurrent $C$. difficile-associated disease by donated stool transplanted via colonoscopy: a case series of 12 patients. $J$ Clin Gastroenterol. 2010;44:562-6.

42. MacConnachie AA, Fox R, Kennedy DR, Seaton RA. Faecal transplant for recurrent Clostridium difficile-associated diarrhoea: a UK case series. QJM. 2009;102: 781-4.

43. Pettersson GB, Martino D, Blackstone EH, Nowicki ER, Houghtaling P, Sabik F Jr, et al. Advising complex patients who require complex heart operations. J Thorac Cardiovasc Surg. 2013;145:1159-69.e3.

44. Kyne L, Hamel MB, Polavaram R, Kelly CP. Health care costs and mortality associated with nosocomial diarrhea due to Clostridium difficile. Clin Infect Dis. 2002;34:346-53 
APPENDIX E1. VARIABLES CONSIDERED IN THE ANALYSES

\section{Demographic}

Age (years),* gender,* race (white/black/other), * weight $(\mathrm{kg})$, height $(\mathrm{cm})$, weight/height ratio, body surface area $\left(\mathrm{m}^{2}\right)$, body mass index $\left(\mathrm{kg} / \mathrm{m}^{2}\right)$.* $^{*}$

\section{Symptoms and Status}

New York Heart Association functional class (I-IV), * emergency operation, ${ }^{*}$ hospital transfer.*

\section{Cardiac Comorbidity}

Previous myocardial infarction, ${ }^{*}$ atrial fibrillation, ${ }^{*}$ complete heart block or pacer, ventricular arrhythmia, heart failure, ${ }^{*}$ endocarditis, ${ }^{*}$ coronary artery disease (number of systems $\geq 50 \%$ ), * previous cardiac operation, $*$ surgery number.

\section{Noncardiac Comorbidity}

Peripheral arterial disease, ${ }^{*}$ carotid disease, ${ }^{*}$ hypertension, ${ }^{*}$ pharmacologically treated diabetes, $*$ insulin-treated diabetes, ${ }^{*}$ non-insulin-dependent diabetes, chronic obstructive pulmonary disease, ${ }^{*}$ history of smoking, ${ }^{*}$ preoperative renal dialysis, ${ }^{*}$ previous stroke, ${ }^{*}$ creatinine $(\mathrm{mg} / \mathrm{dL}),{ }^{*}$ blood urea nitrogen $(\mathrm{mg} / \mathrm{dL})$, bilirubin $(\mathrm{mg} / \mathrm{dL}), *$ creatinine clearance, glomerular filtration rate, hematocrit $(\%), *$ total cholesterol $(\mathrm{mg} / \mathrm{dL})$, high-density lipoprotein cholesterol (mg/dL), * low-density lipoprotein cholesterol $(\mathrm{mg} / \mathrm{dL})$, triglycerides $(\mathrm{mg} / \mathrm{dL})$.

\section{Experience}

Date of operation (years since January 1, 2005), * surgeon.*

\section{Procedural}

Coronary artery bypass grafting, ${ }^{*}$ number of internal thoracic arteries used, ${ }^{*}$ aortic valve procedure, ${ }^{*}$ mitral valve procedure, * tricuspid valve procedure,* aortic procedure, septal myectomy, ${ }^{*}$ mechanical assist device, ${ }^{*}$ transplant, ${ }^{*}$ surgical invasiveness (full, $*$ minimal, percutaneous/ports).

\section{Support}

On-pump,* cardiopulmonary bypass time (minutes),* aortic clamp used, ${ }^{*}$ aortic clamp time (minutes), circulatory arrest, ${ }^{*}$ circulatory arrest time (minutes), total operating room time (minutes).*

\section{Blood Use}

Intraoperative: red blood cell units, platelets, fresh frozen plasma, cryoprecipitate, any intraoperative blood use. Postoperative: red blood cell units, ${ }^{*}$ platelets, fresh frozen plasma, cryoprecipitate, any postoperative blood use. Total: red blood cell units,* platelets, fresh frozen plasma, cryoprecipitate, any intraoperative or postoperative blood use.*

* Variables used in the propensity model. 


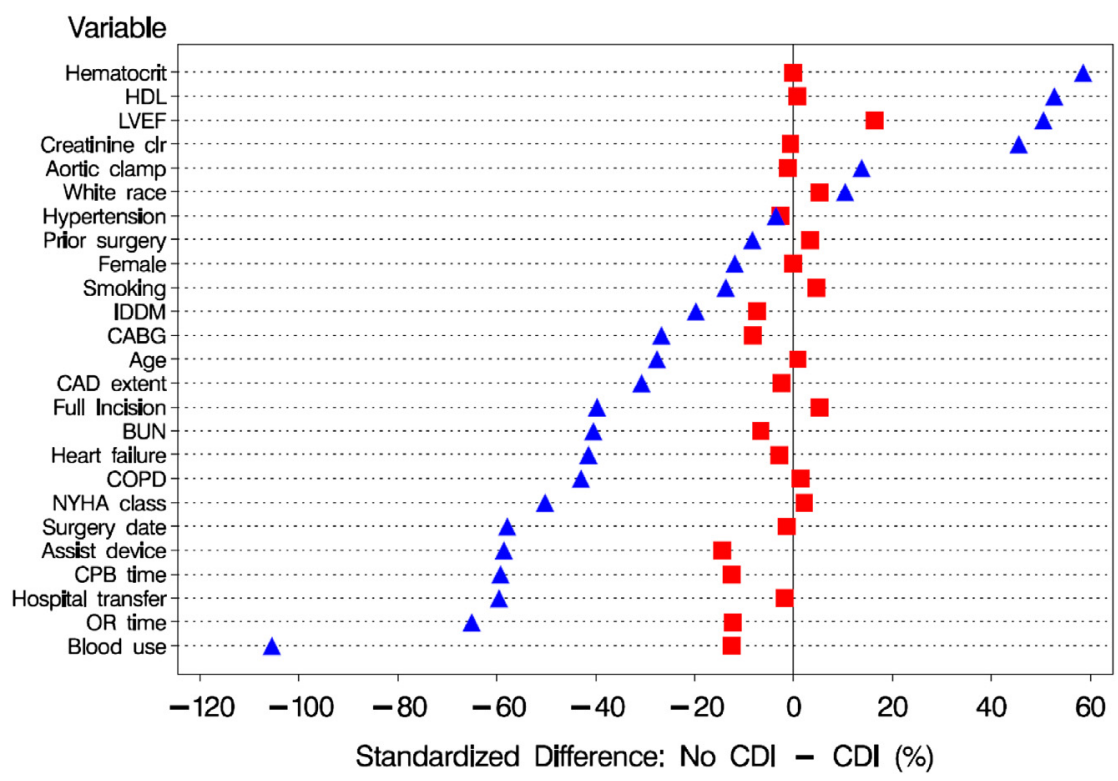

FIGURE E1. Covariable balance description before (triangles) and after (squares) matching between the no CDI and CDI groups.* HDL, High-density lipoprotein; $L V E F$, left ventricular ejection fraction; $c l r$, clearance; $I D D M$, insulin-dependent diabetes; $C A B G$, coronary artery bypass grafting; $C A D$, coronary artery disease; $B U N$, blood urea nitrogen; $C O P D$, chronic obstructive pulmonary disease; $N Y H A$, New York Heart Association; $C P B$, cardiopulmonary bypass; OR, operating room; CDI, Clostridium difficile infection. *Austin PC, Mamdani MM. A comparison of propensity score methods: a case study estimating the effectiveness of post-AMI statin use. Stat Med. 2006;25:2084-106.

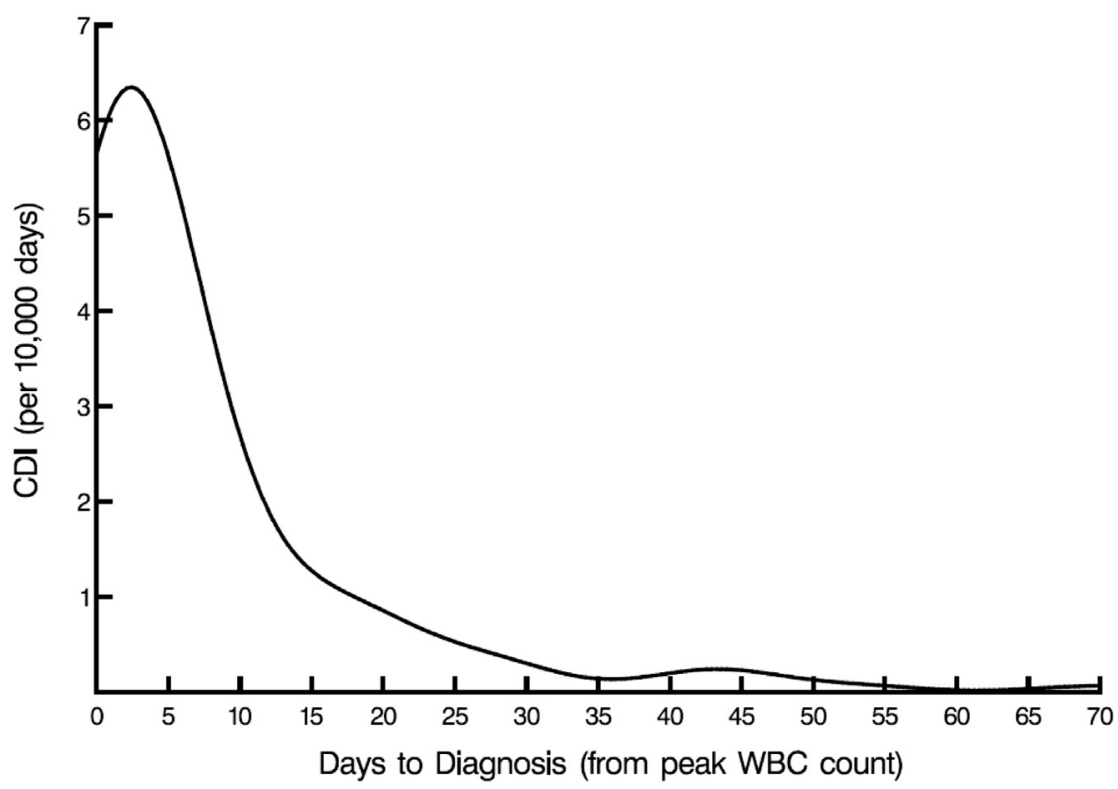

FIGURE E2. Characterization of the onset of Clostridium difficile infection (CDI) after cardiac surgery. Days between peak white blood cell (WBC) count and diagnosis of CDI. 


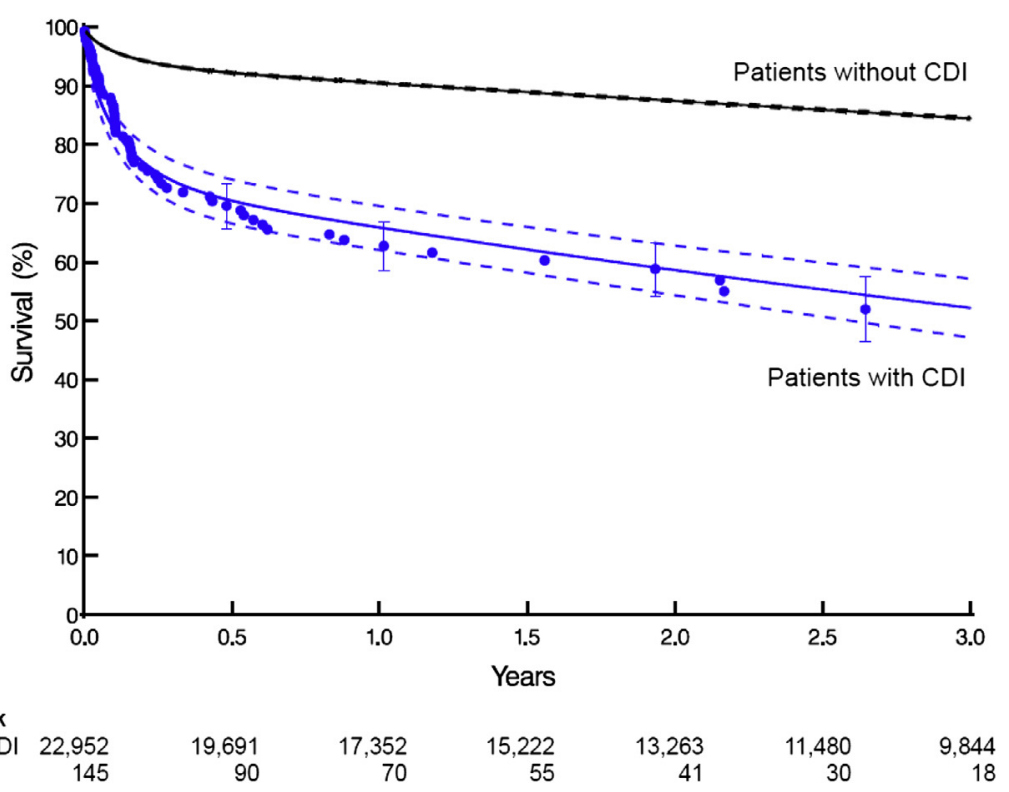

FIGURE E3. Unadjusted survival stratified by whether or not the patient had a Clostridium difficile infection (CDI). Time zero for the upper curve is the date of surgery, with patients censored at occurrence of CDI. Time zero for the lower curve is the time of the CDI diagnosis. Each symbol on the lower curve represents a death positioned on the vertical axis by the Kaplan-Meier estimator, and vertical bars are confidence limits equivalent to \pm 1 standard error. The solid line for both curves is parametric survival enclosed within a dashed $68 \%$ confidence band. The number of patients at risk is given below the horizontal axis. 
A
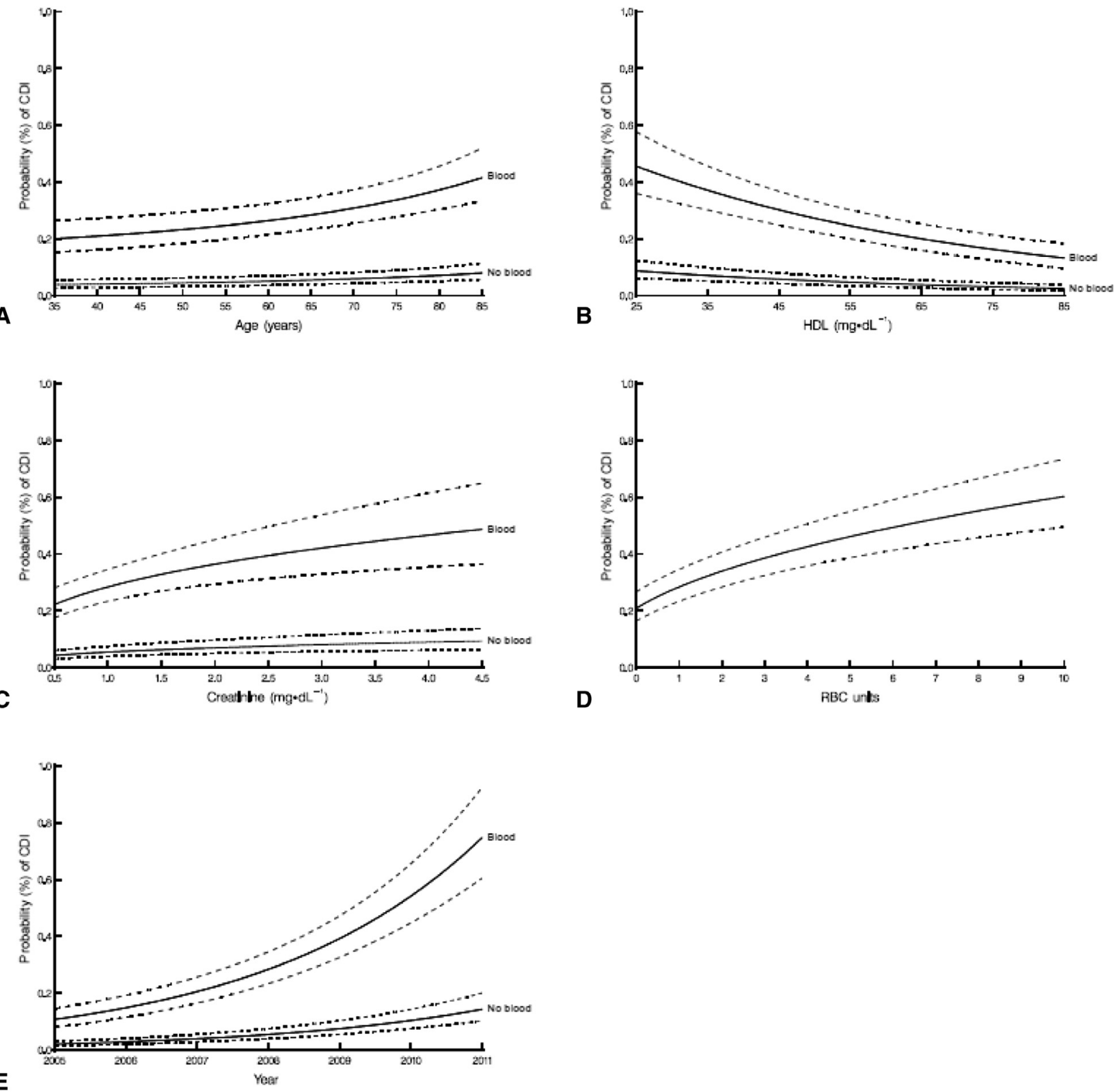

FIGURE E4. Effect of continuous risk factors on the probability of developing Clostridium difficile $(C D I)$ infection using nomograms of the logistic regression equation. Solid lines are parametric estimates enclosed within a $68 \%$ confidence band. Simulations are based on the logistic model (Table 3) for a 65 year-old patient with hypertension, no chronic obstructive pulmonary disease, high-density lipoprotein $(H D L)$ level $48 \mathrm{mg} / \mathrm{dL}$, creatinine level $1.0 \mathrm{mg} / \mathrm{dL}$, no hospital transfer, and no ventricular assist device, stratified by use of blood products. A, Age at operation. B, Preoperative HDL level. C, Preoperative creatinine level. D, Red blood cell $(R B C)$ units transfused. E, Date of operation. 
TABLE E1. Hospital-wide occurrence of Clostridium difficile infection per 10,000 patient-days

\begin{tabular}{cccc}
\hline Year & Number & Patient-days & Rate per 10,000 patient-days \\
\hline 2006 & 203 & 332,119 & 6.11 \\
2007 & 191 & 333,758 & 5.72 \\
2008 & 209 & 339,221 & 6.16 \\
2009 & 204 & 356,612 & 5.72 \\
2010 & 264 & 348,522 & 7.57 \\
2011 & 409 & 343,073 & 11.92 \\
2012 & 368 & 361,681 & 10.17 \\
2013 & 317 & 358,587 & 8.84 \\
\hline
\end{tabular}

